\section{Kommentar}

Die vorliegende retrospektive Arbeit ist auch deshalb ein wichtiger Beitrag zur Diskussion, weil sie Hinweise darauf liefert, welche asymptomatische Karotisstenose möglicherweise nicht operiert werden sollte.

Sie zeigt, dass die fortschreitende Stenosierung und drohende Okklusion allein nur mit einem insgesamt sehr geringen Schlaganfallrisiko assoziiert ist, das unter den peri- prozeduralen Komplikationsraten einer revaskularisierenden Behandlung anzusiedeln ist.

Eine zukünftige individuelle Risikostratifizierung muss vielmehr auf andere Parameter (z. B. Mikroembolie-Detektion, aufgehobene zerebrovaskuläre Reservekapazität, Charakterisierung der Plaquemorphologie, Coated-platelets-Level) als den Stenosegrad allein setzen, um Patienten zu identifizieren, die möglicherweise trotz einer opti- malen medikamentösen Therapie von einer Intervention profitieren könnten.

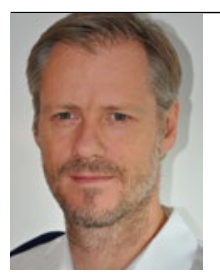

Alexander Correll

Neurologie,

Bundeswehrkranken-

haus Berlin

\title{
Kalorienreduktion nur an zwei Tagen pro Woche: Auch so funktioniert Gewichtsabnahme
}

\begin{abstract}
Wer erfolgreich Gewicht abnehmen will, muss nicht nur das Richtige essen, sondern auch sein Essverhalten optimieren. Ein ungeklärte Frage ist dabei: Soll man täglich wenig essen oder reicht es, nur alle paar Tage die Kalorien reduzieren, um ein Energiedefizit zu erzielen?
\end{abstract}

U $\mathrm{m}$ diese Frage zu beantworten, wurde ein differenziertes Studiendesign bei 115 Frauen im Alter von 20 - 69 Jahren mit einem Body-Mass-Index (BMI) von $24-45 \mathrm{~kg} / \mathrm{m}^{2}$ gewählt: Eine Gruppe hielt täglich eine Reduktionsdiät (TR) als mediterrane Kost mit viel Gemüse, Obst, Vollkorngetreide, Olivenöl, Fisch, Geflügel und magerem Fleisch ein (45\% Kohlenhydrate, $25 \%$ Eiweiß, 30\% Fett). Eine zweite Gruppe nahm intermittierend (IR) eine Kost mit einem Energiedefizit von $70 \%$ an zwei Tagen in der Woche mit 20\% Kohlenhydraten, $45 \%$ Eiweiß und $35 \%$ Fett zu sich. An den restlichen fünf Tagen aßen sie ebenfalls eine mediterrane Kost, jedoch ohne Energiedefizit (eukalorisch). Die dritte Gruppe ernährte sich ähnlich intermittierend hypokalorisch wie die zweite Gruppe, Eiweiß und Fett (IR+EF) waren jedoch nicht restringiert $(45-50 \%$ Kohlenhydrate, 20 - $25 \%$ Eiweiß, $30 \%$ Fett). Es gab somit eine Gruppe mit täglicher hypokalorischer Kost (25\% Energiedefizit) und zwei mit intermittierender hypokalorischer Kost (2 Tage/Woche 70\% Energiedefizit, 5 Tage/Woche eukalorische Ernährung).

Das Ziel, mindestens $5 \mathrm{~kg}$ in drei Monaten abzunehmen, hatten in der Gruppe mit täglicher Restriktion (TR) $48 \%$ der Frauen erreicht, in den anderen bei- den Gruppen mit intermittierender Reduktionskost $58 \%$ (IR+EF) bzw. $65 \%$ (IR). Die korrespondierende Abnahme des Körperfetts betrug 2,0 kg, 3,2 kg und $3,7 \mathrm{~kg}$, und der Taillenumfang nahm um $3,3 \mathrm{~cm}, 4,8 \mathrm{~cm}$ und $5,3 \mathrm{~cm}$ ab. Hinsichtlich der fettfreien Masse (Muskelmasse) gab es keine Gruppenunterschiede. Von den metabolischen Größen waren Parameter der Insulinresistenz (Plasma-Insulin, HOMA-Index) unter der intermittierenden Reduktionsdiät deutlich gebessert, unter einer täglichen Diät zeigte sich dagegen keine Änderung.

Harvie $M$ et al. The effect of intermittent energy and carbohydrate restriction v. daily energy restriction on weight loss and metabolic disease risk markers in overweight women. Br J Nutr. 2013; 110(8): 1534-1547

\section{Kommentar}

Dass eine intermittierende Reduktionskost an zwei Tagen pro Woche mit erheblichem Energiedefizit einer täglichen Reduktionskost mit mäßigem Energiedefizit hinsichtlich einer Verminderung des Körperfetts und Besserung der Insulinresistenz überlegen ist, überrascht. Frühere Studien dieser Art konnten ein solches Ergebnis nicht vorweisen. Der Schlüssel zum Erfolg liegt, wie die Autoren ausführen, wahrscheinlich weniger in der Zusammensetzung der empfohlenen Kost als vielmehr in der Ein-

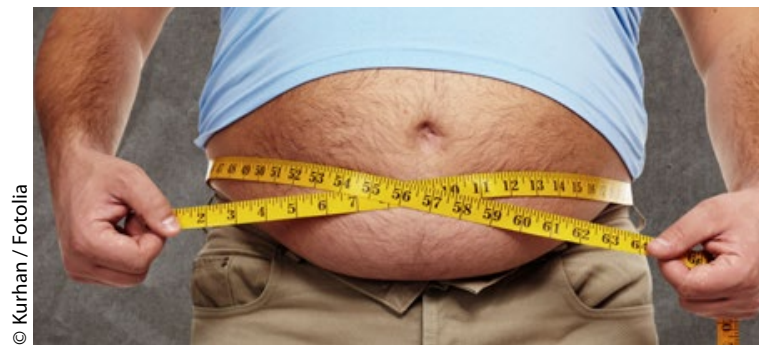

haltung. Für die intermittierende Ernährung wurde eine mediterrane Kost gewählt, die in der Regel von Probanden geschmacklich gut toleriert wird und die durch den relativ hohen Anteil an Ballaststoffen ausreichend sättigt, auch bei kleineren Essportionen. Der vermehrte Konsum von Eiweiß und Fett in einer mediterranen Kost hatte jedoch keinen Vorteil, wenngleich Eiweiß verstärkt sättigt. Fett jedoch kann wegen der Geschmacksverstärkung zu einer vermehrten Energieaufnahme führen. Pathophysiologisch ungeklärt ist, ob eine starke Energierestriktion an wenigen Tagen metabolische Mechanismen induziert, die bei mäßiger Energierestriktion an allen Tagen nicht oder nicht so ausgeprägt in Gang gesetzt werden. Aufgrund von Tierversuchen geht es dabei u.a. um den Intermediärstoffwechsel, Karzinome sowie die Mortalität.

Fazit für die Praxis: Wem es leichter fällt, an zwei Tagen in der Woche die Nahrungsaufnahme erheblich zu vermindern statt die ganze Woche weniger zu essen, der soll dieses Regime praktizieren.

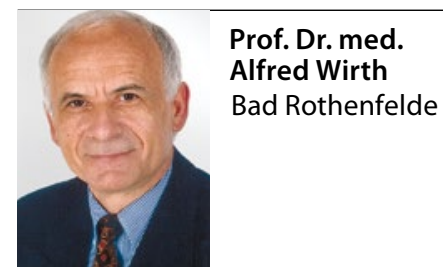

